\title{
The immunophenotypic spectrum of primary mediastinal large B-cell lymphoma reveals prognostic biomarkers associated with outcome
}

Jacob R. Bledsoe, MD, ${ }^{1}$ Robert A. Redd, MS, ${ }^{2}$ Robert P. Hasserjian, MD,${ }^{1}$ Jacob D. Soumerai, MD, ${ }^{3}$ Ha T. Nishino, MD, ${ }^{4}$ Daniel F. Boyer, MD, PhD, ${ }^{5}$ Judith A. Ferry, MD, ${ }^{1}$ Lawrence R. Zukerberg, MD, ${ }^{1}$ Nancy Lee Harris, MD, ${ }^{1}$ Jeremy S. Abramson, MD, ${ }^{6}$ and Aliyah R. Sohani, $\mathrm{MD}^{1}$

${ }^{1}$ Department of Pathology, Massachusetts General Hospital and Harvard Medical School, Boston, MA, USA;

${ }^{2}$ Department of Biostatistics and Computational Biology, Dana-Farber Cancer Institute, Boston, MA, USA;

${ }^{3}$ Lymphoma Service, Memorial Sloan Kettering Cancer Center, New York, NY, USA;

${ }^{4}$ Department of Pathology, North Shore Medical Center, Salem, MA, USA;

${ }^{5}$ Department of Pathology, University of Michigan, Ann Arbor, MI, USA;

${ }^{6}$ Center for Lymphoma, Massachusetts General Hospital Cancer Center and Department of

Medicine, Harvard Medical School, Boston, MA, USA

\section{Author for correspondence:}

Aliyah R. Sohani MD

Department of Pathology

Massachusetts General Hospital

55 Fruit St., WRN 219

Boston, MA 02114

Phone: (617) 726-3187

Fax : (617) 726-7474

E-mail: arsohani@partners.org

\section{Manuscript information:}

Abstract word count: 248

Manuscript text word count: 3362

Tables: 2

Figures: 2

Supplemental files: 1

Running title: Immunophenotypic prognostic markers in PMBL

$\underline{\text { Keywords: }}$ primary mediastinal large B-cell lymphoma, immunophenotype, prognosis, PDL1, MUM1

This is the author manuscript accepted for publication and has undergone full peer review but has not been through the copyediting, typesetting, pagination and proofreading process, which may lead to differences between this version and the Version record. Please cite this article as doi:10.1002/ ajh.24485. 


\section{Abstract}

Primary mediastinal large B-cell lymphoma (PMBL) is a distinct subtype of diffuse large Bcell lymphoma (DLBCL) that shows overlap with classical Hodgkin lymphoma (CHL) and a favorable prognosis compared to mediastinal gray-zone lymphoma (MGZL). We performed immunohistochemistry on initial diagnostic specimens of 49 cases of uniformly-treated PMBL to determine the frequency and clinical significance of expression of antigens commonly seen in CHL and MGZL, along with markers previously shown to be prognostic in DLBCL, not otherwise specified. The median age was 37 years with a female:male ratio of 2.3. After a median follow-up of 78 months, $24 \%$ of patients had relapsed or refractory disease and 22\% had died; the 5-year PFS was 70\%. Variable CD15 expression was seen in $31 \%$ of cases, but was not associated with adverse outcome. Hans cell-of-origin, proliferation index, and MYC/BCL2 coexpression were not associated with outcome, while low PDL1 ( $p=0.011)$ and high MUM1 $(p=0.065)$ staining were each associated with shorter PFS. A biologic risk score (one point each for low PDL1 and high MUM1) stratified patients into three prognostic risk groups for PFS $(p=0.001)$ and OS $(p=0.032)$. On separate multivariate models, low PDL1 was independent of R-IPI risk group for PFS (HR 6.0, $\mathrm{p}=0.023$ ), as was a biologic risk score of 2 (HR 5.6, $\mathrm{p}=0.011$ ). Incorporation of the biologic risk score sub-stratified patients within R-IPI groups for both PFS $(\mathrm{p}<0.001)$ and OS $(p<0.001)$. In summary, we characterize the immunophenotypic spectrum of PMBL and identify PDL1 and MUM1 as prognostic biomarkers for high-risk disease. 


\section{Introduction}

Primary mediastinal (thymic) large B-cell lymphoma (PMBL) is a subtype of diffuse large B-cell lymphoma (DLBCL) with distinct clinicopathologic and genetic features. It accounts for $2-4 \%$ of non-Hodgkin lymphoma and occurs predominantly in young adults with a median age of 35 years and a female predominance (male:female ratio of 1:2) (1). Patients typically present with a bulky mediastinal mass that frequently invades adjacent structures, while involvement of bone marrow or non-mediastinal lymph nodes is uncommon at diagnosis $(1,2)$.

PMBL shares a number of features with classical Hodgkin lymphoma (CHL), particularly the nodular sclerosis type. Cases of PMBL often exhibit fibrosis and may contain cells with pleomorphic, multilobated nuclei resembling Reed-Sternberg cells and variants, and neoplastic cells often express CD30 similar to Reed-Sternberg cells (1). Occasionally, CHL and PMBL have been reported together as composite lymphomas or as metachronous tumors in the same patient (3). Therefore, it is not surprising that PMBL shares a genetic transcription profile with CHL that is distinct from that of DLBCL, not otherwise specified (DLBCL-NOS), with activation of the NFKB antiapoptotic survival pathway and the JAK-STAT signaling pathway (4, 5). Combined expression of the NFKB targets, c-rel and TRAF1, has been found by immunohistochemistry in more than $80 \%$ of CHL and $50 \%$ of PMBL, but only rarely in DLBCL-NOS $(6,7)$. Amplifications in 9p24 resulting in increased JAK-STAT activity and upregulation of $P D L 1$ and $P D L 2$, and translocations of CIITA at $16 \mathrm{p} 13$ are common in both CHL and PMBL; these aberrations involving PDL1, PDL2 and CIITA have been implicated in tumormediated immune evasion in both entities $(8,9)$. More recently, somatic mutations involving 
PTPN1 have been identified in both CHL and PMBL, further supporting a biological overlap (10). A consequence of this overlap is the existence of cases with morphologic and immunophenotypic features intermediate between PMBL and CHL and with shared genetic features, termed mediastinal gray-zone lymphoma (MGZL), which is clinically more aggressive than either PMBL or CHL (3, 11-16).

The clinical outcome in PMBL appears favorable in comparison to typical DLBCL, but this may reflect the tendency of these patients to present with lower-risk features such as young age and limited stage (4). The most commonly employed first-line treatment regimen for PMBL historically has been R-CHOP (rituximab, cyclophosphamide, doxorubicin, vincristine, and prednisone), usually combined with radiation therapy due to the bulky, localized nature of this disease. However, multiple retrospective studies in the pre-rituximab era suggested that CHOP is inferior to more intensive regimens in PMBL, and only limited retrospective and prospective data in the modern era support the benefit of rituximab when added to CHOP chemotherapy (1722). In addition, a recent retrospective analysis from the Massachusetts General Hospital (MGH) of 63 adult PMBL patients treated with $\mathrm{R}-\mathrm{CHOP}+/$ - radiation therapy demonstrated a high rate of primary refractory disease (23). Similar high rates of treatment failure have been reported in pediatric patients (12). Unfortunately, the cure rate for progressive disease after initial treatment is low. A recent prospective study of dose-adjusted R-EPOCH (rituximab, etoposide, prednisone, vincristine, cyclophosphamide, and doxorubicin) was associated with a high response rate in PMBL (24), but this intense regimen may be unnecessary in some patients. These findings point to the need for improved prognostic biomarkers in PMBL, in order to identify patients who may benefit from higher intensity regimens or novel treatment approaches. 
We have encountered occasional cases of morphologically typical PMBL that express antigens commonly seen in CHL or MGZL, such as CD15, but that do not fulfill 2008 WHO criteria for a diagnosis of MGZL (16). The prevalence and clinical significance of such findings in PMBL is unknown. Therefore, we sought to investigate the prognostic significance of expression of a CHL or MGZL-like immunophenotype in PMBL, along with the significance of expression of antigens related to cell-of-origin, proliferation, apoptosis, and immune evasion that have previously been shown to have prognostic significance in DLBCL-NOS (25-28), with a focus on antibodies routinely available for clinical diagnostic use.

\section{Methods \\ Case Selection}

Following Partners HealthCare Institutional Review Board approval with waiver of patient consent (protocol \# 2007P001458, last approval 1/25/2016), we performed a computer-assisted search of the MGH pathology files and clinicopathologic database of hematologic malignancies for all cases of PMBL treated with R-CHOP with or without radiation therapy. Patients were identified based on a combination of clinical and pathological criteria, including a large B-cell lymphoma presenting in the anterior mediastinum as the dominant site of disease with typical pathological features of PMBL on tissue biopsy (1). Only patients who had completed therapy and with sufficient pre-treatment biopsy tissue for tissue microarray (TMA) construction and/or further immunohistochemical analysis were included, yielding a total of 49 cases diagnosed 
between 1996 and 2012. Of these, 41 patients had been included in a prior study in which clinical features and outcome, but not correlation with histopathologic features, were previously reported (23); however, inclusion in the prior analysis was not a selection criterion for inclusion in the current study.

\section{TMA Construction and Immunohistochemical Analysis}<smiles>C1CCC1</smiles>

TMAs were manually constructed using $2 \mathrm{~mm}$ diameter tissue cores of tumor cell-rich representative areas of lymphoma in cases with sufficient tissue, with inclusion of cores of reactive lymphoid control tissue. In five cases with limited tissue, selected immunohistochemical stains were performed on whole-tissue sections and evaluated in tumor cell-rich areas. Immunohistochemistry was performed on $5 \mu \mathrm{m}$ formalin- or B-plus-fixed, paraffin-embedded tissue sections using an automated immunostainer (BOND-III, Leica Biosystems, Buffalo Grove, IL). Briefly, sections were deparaffinized, antigen retrieval was performed with an EDTA-based solution (Leica) at $\mathrm{pH}$ 9, and sections were stained with antibodies to pan-B-cell antigens, CHLassociated antigens, and antigens previously shown to have prognostic significance in DLBCLNOS (Supplemental Table 1). All of the antibodies used in the study are available at our institution for clinical diagnosis and have been validated for both formalin and B-plus fixatives with comparable staining results.

Immunohistochemical stains were evaluated independently by two authors (JRB and ARS) who were blinded to patient outcome, with resolution of scoring discrepancies via consensus review. For MYC and Ki67, percent staining of neoplastic lymphoid cells was estimated in deciles. PD1 
staining of background small lymphocytes was scored as positive or negative, with any staining considered positive. For all other antibodies, scores were assigned for staining intensity $(0=$ no staining, $1+=$ weak staining, $2+=$ moderate staining, $3+=$ strong staining) and percentage of lymphoma cells staining (0: $0 \%, 1+: 1-25 \%, 2+: 26-50 \%, 3+: 51-75 \%, 4+:>75 \%)$. Staining intensity and frequency scores were multiplied to yield an overall $\mathrm{H}$-score ranging from $0-12$, which was categorized as follows: no staining (0), weak/focal or low overall staining (1-3) and moderate/strong/diffuse or high overall staining (4-12). Interobserver agreement for antibody staining scores was overall high $(\kappa=0.97,95 \%$ confidence interval [CI]: 0.96-0.98).

Statistical Analysis

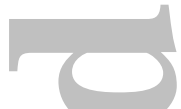

Descriptive statistics were used to characterize patients at baseline. Ann Arbor stage was determined from baseline radiology reports or from documentation in the medical record by the primary oncologist. Tumor characteristics, including type and number of extranodal sites of disease, were included in the analysis only where documented in baseline radiology reports. Primary endpoints were overall survival (OS), defined as time from initial diagnosis until death from any cause, censoring at time last known alive for patients still alive, and progression-free survival (PFS), defined as time from initial diagnosis until disease progression or death, censoring at time last known alive and disease-free for patients alive without progressive disease. OS and PFS curves were obtained using the Kaplan-Meier method with 95\% confidence intervals calculated using Greenwood's formula. Univariate analysis was performed to assess predictors of outcome and tested for significance using the log-rank test. Multivariable Cox proportional hazards models were used to estimate hazard ratios with confidence intervals. 
Univariate and multivariable recursive partitioning classification analyses were performed to identify significant prognostic factors for time-to-event outcomes. Wilcoxon rank sum tests were used for comparison of continuous variables. Fisher's exact tests were used for comparison of categorical variables. Interobserver agreement was quantified by Cohen's kappa coefficient. A two-sided alpha error of 0.05 was used for all statistical tests, and statistical analyses were performed using $\mathrm{R}$ version 3.1.2.

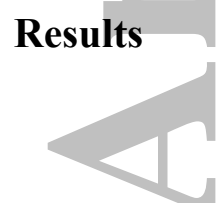

Clinical Characteristics and Outcome

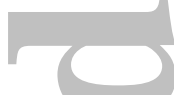

Clinical features of the 49 patients at the time of initial diagnosis are summarized in Table I and detailed in Supplemental Table 2. The median age was 37 years (range 19-82), with a male:female ratio of 1:2.3. Since many patients had been included in the previously described cohort of 63 patients (23), we found similar outcomes to that study including a high rate of primary induction failure, with seven cases with progressive disease during chemotherapy (14\%) and two with treatment-related mortality (4\%). After an overall median follow up of 78 months, 12 patients $(24 \%)$ had relapsed or refractory disease (median 5.9 months after diagnosis) and 11 (22\%) had died (median 15.9 months after diagnosis). The 5-year PFS was 70\% (95\% CI: 55$81 \%$ ), and the 5-year OS was 76\% (95\% CI 60-86\%). Longer PFS was associated with limited stage disease $(\mathrm{p}<0.001)$, low/intermediate Revised International Prognostic Index $(\mathrm{R}-\mathrm{IPI})$ score of $0-2(p<0.001$, Figure $2 C)$, and female sex $(p=0.039)$. Other clinical factors, including bulky 
disease (mediastinal mass size $>10 \mathrm{~cm}$ ) and administration of radiotherapy, were not associated with outcome.

\section{Immunophenotypic Features}

The histopathologic features of the 49 cases are summarized in Table II and detailed in Supplemental Table 3. All cases expressed CD45, CD19 and CD20, although occasional cases $(2-6 \%)$ showed low expression of one of these markers (Figure 1A-B). Other pan-B-cell markers, including BOB.1, CD22, CD79a, OCT2 and PAX5, were widely expressed, with a subset of cases showing low overall expression and rare cases (2-4\%) showing no expression of one or more of these markers. CD23 expression was present in $66 \%$ of cases (Figure 1C). CD30 was expressed in $78 \%$ of cases, with approximately half of cases showing high staining and the remainder showing low overall staining (Figure 1D). CD15 was weakly (1+ intensity score) and/or focally (1+ frequency score) expressed by tumor cells, yielding low overall staining in $25 \%$ cases, and showed high overall staining by tumor cells in three cases $(6 \%)$, typically in a paranuclear dot-like pattern (Figure 1E-F). Such expression was confirmed to be on tumor cells, and not on background macrophages, by examination under 100x magnification and determining the positively-stained cells to be large lymphoid cells based on their morphology on hematoxylin counterstain (Figure 1E inset). Ten cases demonstrated both low overall CD15 expression and some degree of staining for CD30 (7 high and 3 low overall CD30 expression). Of the three cases with high overall CD15 staining, two showed low and one showed high overall coexpression of CD30, while all three exhibited high staining of the pan-B-cell markers CD19, CD20 and PAX5. Only 26\% of cases were positive for CD10, while most cases (93\%) expressed 
MUM1. High overall BCL2 expression was present in 64\% of cases, but MYC/BCL2 doubleexpression, using cutoffs of $\geq 40 \%$ for MYC and $\geq 50 \%$ for BCL2 as previously described (27), was present in only six cases (14\%). PDL1 was expressed by tumor cells in $71 \%$ of cases. PD1 was weakly and focally expressed by microenvironmental tumor-infiltrating lymphocytes in only two cases $(5 \%)$. In cases lacking PDL1 expression on tumor cells, some showed staining of rare scattered background immune cells but significant microenvironmental PDL1 expression was not seen. Flow cytometry results were available for 15 cases $(31 \%)$, eight of which $(53 \%)$ demonstrated monoclonal surface immunoglobulin light chain expression, while the remainder lacked surface immunoglobulin expression.

Association of Immunohistochemical Features with Outcome

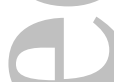

On univariate analysis, high overall PDL1 staining (H-score 4-12) was associated with longer PFS ( $\mathrm{p}=0.011)$, while negative/low overall MUM1 staining (H-score 0-3) was marginally associated with longer PFS ( $\mathrm{p}=0.065)$ (Figures $1 \mathrm{G}-\mathrm{L}$ and $2 \mathrm{~A})$. The association between PDL1 or MUM1 expression and OS did not reach statistical significance $(\mathrm{p}>0.05)$. Expression of CD15, CD30, Ki67, MYC, combined MYC/BCL2 double-expression, and Hans classifier cell-of-origin were also not associated with outcome in terms of staining intensity, frequency or overall score. Dual expression of CD15 and CD30 was not associated with outcome.

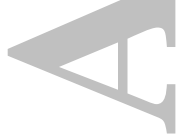

We developed a biologic risk score by combining the two biomarkers whose overall staining scores were most strongly associated with PFS on univariate analysis, assigning one point each for negative/low PDL1 and high MUM1 overall staining, both factors associated with increased 
risk. The combined PDL1/MUM1 biologic risk score stratified the 46 patients for whom results of both stains were available into three prognostic groups for PFS $(p=0.001)$ and OS $(p=0.032)$ : patients with scores of 0 and 1 had better survival (median PFS not reached) vs. those with a score of 2 (median PFS 28 months) (Figure 2B). On multivariable Cox proportional hazards model analysis (Supplemental Table 4), negative/low PDL1 staining was independent of the RIPI risk group for PFS (hazard ratio [HR] 6.0, $\mathrm{p}=0.023,95 \%$ CI 1.3-27.9). In a separate model, the combined PDL1/MUM1 biologic risk score and R-IPI were independent predictors for PFS: biologic risk score of 2 vs. 0-1, HR 5.6 ( $\mathrm{p}=0.011,95 \%$ CI 1.5-21.5) and R-IPI $>2$ vs. 0-2, HR 9.9 $(\mathrm{p}<0.001,95 \%$ CI $2.7-36.0)$.

Incorporation of the biologic risk score into traditional clinical risk stratification showed the biologic risk score to be prognostic within R-IPI groups for both PFS ( $p<0.001$, Figure 2D) and OS ( $p<0.001$, data not shown). Within the low to intermediate $(0-2)$ R-IPI group that included the majority of patients ( $85 \%$ ), those with a biologic risk score of 2 had an inferior PFS at 5 years $(58 \%, 95 \%$ CI $27-80 \%)$ vs. those with a biologic risk score of $0-1(92 \%, 95 \%$ CI $70-98 \%)$ $(p=0.012)$. Among the seven patients with R-IPI $>2$, five $(71 \%)$ had a biologic risk score of 2 , all of whom had died within a year of diagnosis, while one of the two patients with a biologic risk score of $0-1$ remains alive at two years.

\section{Discussion}

We performed a detailed immunohistochemical characterization of a large group of PMBL and identified an immunophenotypic spectrum within this DLBCL subtype, including variable CD15 
expression in a subset of cases. Correlation of immunophenotypic features with outcome in our uniformly-treated cohort showed that prognostic biomarkers traditionally used in DLBCL-NOS have limited relevance in PMBL and that CD15 expression in otherwise typical PMBL does not portend an adverse outcome. Finally, we developed a novel biologic risk score for PMBL, incorporating staining for MUM1 and PDL1, that appears to stratify patients within traditional RIPI subgroups and that may have clinical utility in identifying high-risk patients.

Our findings largely reflect the immunohistochemical profile previously described in PMBL, including retained pan-B-cell antigen expression in almost all cases and frequent expression of CD23, CD30, MUM1 and PDL1 $(1,29,30)$. Nearly one-third of cases showed some degree of CD15 expression in tumor cells, a feature thought to be uncommon in PMBL $(1,31)$. Our findings suggest that weak or focal CD15 staining may be more frequent in PMBL than previously thought, and that occasional cases of otherwise typical PMBL (e.g., those with DLBCL-like morphology and retained pan-B-cell antigen expression) may show moderate or even strong CD15 expression that is typically in a paranuclear dot-like pattern. However, CD15 expression in our PMBL cohort, both alone and in combination with CD30 expression, was not associated with adverse clinical outcome, in contrast to what has been suggested for MGZL with CD15 expression (31). Our findings point to the need for employing strict criteria in rendering a diagnosis of MGZL, and suggest that cases of otherwise typical PMBL with CD15 co-expression should not be diagnosed as MGZL, unless additional features of CHL-like morphology or pan-Bcell antigen loss are present. 
We studied a number of immunohistochemical markers previously found to be prognostic in DLBCL-NOS, including germinal center vs. non-germinal center cell-of-origin (25), Ki67 proliferation index (32), high MYC expression (33), and double-expression of MYC and BCL2 $(27,34)$, using commercial antibodies routinely available for clinical diagnostic use. None of these biomarkers was associated with outcome in our series, suggesting that immunohistochemical prognostic markers of DLBCL-NOS are not applicable to PMBL. We identified two potential PMBL-specific biomarkers, low PDL1 and high MUM1 expression, as being most strongly associated with an adverse outcome on univariate analysis. By combining both biomarkers into a PDL1/MUM1 biologic risk score ranging from 0-2, we were able to stratify patients into three prognostic groups with good, intermediate and poor survival and substratify patients within the low to intermediate R-IPI subgroup. The biologic risk score retained its significance independently of the R-IPI on multivariate analysis for PFS. Our findings suggest that immunohistochemical evaluation of PDL1 and MUM1 at the time of initial PMBL diagnosis may enhance traditional clinical risk stratification and may be useful to prognosticate and predict response to R-CHOP chemotherapy, as well as to identify patients who may benefit from more intense regimens such as dose-adjusted R-EPOCH. Additional studies are needed to validate these results, particularly in a prospective setting.

High MUM1 expression has been associated with an inferior outcome in DLBCL-NOS, likely due to the role of the MUM1 gene, IRF4, in lymphocyte activation and proliferation in the terminal stages of B-cell differentiation into plasma cells $(25,35,36)$. However, the biologic mechanism by which low PDL1 expression might contribute to an adverse clinical outcome in PMBL is uncertain. PMBL has been associated with a more favorable outcome than DLBCL- 
NOS (4), and PDL1 expression is more frequent in PMBL than in other DLBCL subtypes (28). Therefore, the adverse outcome seen in PMBL with low PDL1 expression may reflect tumor biology more similar to DLBCL-NOS than to classic PMBL.

In many malignancies, high PDL1 expression is associated with a poor prognosis due to its downregulation of the host anti-tumor immune response through interaction with PD1 on effector T cells (37). In one study of a heterogeneous group of DLBCL, including some cases of PMBL, increased PDL1 expression (defined as $\geq 30 \%$ staining of lymphoma cells) was associated with inferior OS (28). However, there is little prior data on the association of PDL1 expression with outcome in a pure cohort of uniformly treated PMBL. One study of 27 cases of non-uniformly treated PMBL found no association between PDL1 staining and outcome using a cutoff of $30 \%$ positivity (38). Similarly, in another study of 51 cases using a cut-off of $5 \%$ positivity, there was no association between PDLI staining and outcome, although treatment data were not reported (39). In contrast to these prior studies, our cohort was restricted to cases of PMBL that were uniformly treated, and other DLBCL subtypes were excluded.

As in PMBL, the Reed-Sternberg cells of CHL demonstrate high PDL1 expression. The tumorinfiltrating T cells in CHL express PD1 with high frequency, providing a rationale for PDL1/PD1-mediated immune escape in CHL and therapeutic PD1 blockade (40, 41). In contrast to $\mathrm{CHL}$, we and others have found PD1 to be infrequently expressed on tumor-infiltrating lymphocytes in PMBL (42), suggesting that PDL1/PD1-mediated immune escape may be less important for tumor cell survival in PMBL than in other malignancies with a higher frequency of PD1-positive background inflammatory cells. Moreover, the role of PDL1 and PD1 in immune 
evasion may differ between tumor types and remains to be fully elucidated in lymphomas.

Indeed, increased numbers of PD1-positive tumor infiltrating lymphocytes have been shown to be a favorable prognostic feature in follicular lymphoma, suggesting that PDL1/PD1 interactions may operate indirectly via inhibition of pro-tumor microenvironmental inflammatory responses in some lymphoma subtypes (42-44).

In summary, we characterize the immunophenotypic spectrum of PMBL and demonstrate that CD15, a marker traditionally associated with MGZL and CHL, may be expressed in a subset of PMBL, although expression is usually weak or focal in most such cases. CD15 expression does not appear to negatively impact prognosis, even in cases where expression is strong or diffuse or when CD30 is co-expressed. Although immunohistochemical markers with prognostic utility in DLBCL-NOS appear to lack relevance in PMBL, we identify PDL1 and MUM1 as two potential PMBL-specific biomarkers. We demonstrate that a biologic risk score incorporating these two stains, both of which are widely available for routine diagnostic use, is independent of clinical risk stratification by R-IPI and can sub-stratify patients within R-IPI groups, particularly those considered low-to-intermediate risk. Additional studies are needed to validate the utility of this biologic risk score in identifying higher-risk patients who may benefit from intensified or novel treatment approaches.

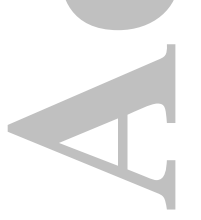




\section{Acknowledgments}

The would like to thank the MGH Histology and Immunohistochemistry Laboratories for their technical expertise in TMA construction and immunohistochemical staining, as well as Dr.

Donna Neuberg for valuable commentary on the manuscript. ARS received salary support from the MGH Department of Pathology and Harvard Medical School through the Eleanor and Miles Shore 50th Anniversary Fellowship Program for Scholars in Medicine.

\section{Authorship Contributions}

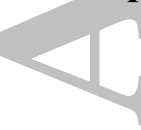

JRB designed the research, collected and analyzed the data, and wrote the manuscript. RAR, RPH, JAF, LRZ and NLH analyzed the data and edited the manuscript. JDS, HTN and DFB collected the data and edited the manuscript. JSA collected and analyzed the data and edited the manuscript. ARS designed the research, collected and analyzed the data, edited the manuscript, and supervised the study.

\section{Disclosure of Conflicts of Interest}

The authors have no relevant conflicts of interest to disclose.

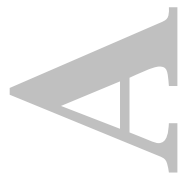




\section{References:}

1. Gaulard P, Harris NL, Pileri SA, et al. Primary mediastinal (thymic) large B-cell lymphoma. In: Swerdlow SH, Campo E, Harris NL, Jaffe ES, Pileri SA, Stein H, Thiele J, Vardiman JW, editors. WHO Classification of Tumours of Haematopoietic and Lymphoid Tissues. Lyon: IARC Press; 2008. pp 250-251.

2. Bishop PC, Wilson WH, Pearson D, et al. CNS involvement in primary mediastinal large B-cell lymphoma. J Clin Oncol 1999;17:2479-2485.

3. Traverse-Glehen A, Pittaluga S, Gaulard P, et al. Mediastinal gray zone lymphoma: the missing link between classic Hodgkin's lymphoma and mediastinal large B-cell lymphoma. Am J Surg Pathol 2005;29:1411-1421.

4. Rosenwald A, Wright G, Leroy K, et al. Molecular diagnosis of primary mediastinal B cell lymphoma identifies a clinically favorable subgroup of diffuse large B cell lymphoma related to Hodgkin lymphoma. J Exp Med 2003;198:851-862.

5. Savage KJ, Monti S, Kutok JL, et al. The molecular signature of mediastinal large B-cell lymphoma differs from that of other diffuse large B-cell lymphomas and shares features with classical Hodgkin lymphoma. Blood 2003;102:3871-3879.

6. Rodig SJ, Savage KJ, LaCasce AS, et al. Expression of TRAF1 and nuclear c-Rel distinguishes primary mediastinal large cell lymphoma from other types of diffuse large B-cell lymphoma. Am J Surg Pathol 2007;31:106-112.

7. Rodig SJ, Savage KJ, Nguyen V, et al. TRAF1 expression and c-Rel activation are useful adjuncts in distinguishing classical Hodgkin lymphoma from a subset of morphologically or immunophenotypically similar lymphomas. Am J Surg Pathol 2005;29:196-203. 
8. Steidl C, Shah SP, Woolcock BW, et al. MHC class II transactivator CIITA is a recurrent gene fusion partner in lymphoid cancers. Nature 2011;471:377-381.

9. Green MR, Monti S, Rodig SJ, et al. Integrative analysis reveals selective 9p24.1 amplification, increased PD-1 ligand expression, and further induction via JAK2 in nodular sclerosing Hodgkin lymphoma and primary mediastinal large B-cell lymphoma. Blood $2010 ; 116: 3268-3277$.

10. Gunawardana J, Chan FC, Telenius A, et al. Recurrent somatic mutations of PTPN1 in primary mediastinal B cell lymphoma and Hodgkin lymphoma. Nat Genet 2014;46:329-335. 11. Grant C, Dunleavy K, Eberle FC, et al. Primary mediastinal large B-cell lymphoma, classic Hodgkin lymphoma presenting in the mediastinum, and mediastinal gray zone lymphoma: what is the oncologist to do? Curr Hematol Malig Rep 2011;6:157-163.

12. Oschlies I, Burkhardt B, Salaverria I, et al. Clinical, pathological and genetic features of primary mediastinal large B-cell lymphomas and mediastinal gray zone lymphomas in children. Haematologica 2011;96:262-268.

13. Evens AM, Kanakry JA, Sehn LH, et al. Gray zone lymphoma with features intermediate between classical Hodgkin lymphoma and diffuse large B-cell lymphoma: characteristics, outcomes, and prognostication among a large multicenter cohort. Am J Hematol 2015;90:778783.

14. Eberle FC, Rodriguez-Canales J, Wei L, et al. Methylation profiling of mediastinal gray zone lymphoma reveals a distinctive signature with elements shared by classical Hodgkin's lymphoma and primary mediastinal large B-cell lymphoma. Haematologica 2011;96:558-566. 15. Eberle FC, Salaverria I, Steidl C, et al. Gray zone lymphoma: chromosomal aberrations with immunophenotypic and clinical correlations. Mod Pathol 2011;24:1586-1597. 
16. Jaffe ES, Stein H, Swerdlow SH, et al. B-cell lymphoma, unclassifiable, with features intermediate between diffuse large B-cell lymphoma and classical Hodgkin lymphoma. In: Swerdlow SH, Campo E, Harris NL, Jaffe ES, Pileri SA, Stein H, Thiele J, Vardiman JW, editors. WHO Classification of Tumours of the Haematopoietic and Lymphoid Tissues. Lyon: IARC Press; 2008. pp 267-268.

17. Rieger M, Osterborg A, Pettengell R, et al. Primary mediastinal B-cell lymphoma treated with CHOP-like chemotherapy with or without rituximab: results of the Mabthera International Trial Group study. Ann Oncol 2011;22:664-670.

18. Savage KJ, Al-Rajhi N, Voss N, et al. Favorable outcome of primary mediastinal large Bcell lymphoma in a single institution: the British Columbia experience. Ann Oncol 2006; 17:123130.

19. Zinzani PL, Martelli M, Bertini M, et al. Induction chemotherapy strategies for primary mediastinal large B-cell lymphoma with sclerosis: a retrospective multinational study on 426 previously untreated patients. Haematologica 2002;87:1258-1264.

20. Vassilakopoulos TP, Pangalis GA, Katsigiannis A, et al. Rituximab, cyclophosphamide, doxorubicin, vincristine, and prednisone with or without radiotherapy in primary mediastinal large B-cell lymphoma: the emerging standard of care. Oncologist 2012;17:239-249.

21. Todeschini G, Secchi S, Morra E, et al. Primary mediastinal large B-cell lymphoma (PMLBCL): long-term results from a retrospective multicentre Italian experience in 138 patients treated with CHOP or MACOP-B/VACOP-B. Br J Cancer 2004;90:372-376.

22. Savage KJ, Yenson PR, Shenkier T, et al. The outcome of primary mediastinal large Bcell lymphoma (PMBCL) in the R-CHOP treatment era [abstract]. Blood 2012;120:303. 
23. Soumerai JD, Hellmann MD, Feng Y, et al. Treatment of primary mediastinal B-cell lymphoma with rituximab, cyclophosphamide, doxorubicin, vincristine and prednisone is associated with a high rate of primary refractory disease. Leuk Lymphoma 2014;55:538-543. 24. Dunleavy K, Pittaluga S, Maeda LS, et al. Dose-adjusted EPOCH-rituximab therapy in primary mediastinal B-cell lymphoma. N Engl J Med 2013;368:1408-1416.

25. Hans CP, Weisenburger DD, Greiner TC, et al. Confirmation of the molecular classification of diffuse large B-cell lymphoma by immunohistochemistry using a tissue microarray. Blood 2004;103:275-282.

26. $\mathrm{Hu} \mathrm{S}, \mathrm{Xu}-\mathrm{Monette} \mathrm{ZY}$, Tzankov A, et al. MYC/BCL2 protein coexpression contributes to the inferior survival of activated B-cell subtype of diffuse large B-cell lymphoma and demonstrates high-risk gene expression signatures: a report from The International DLBCL Rituximab-CHOP Consortium Program. Blood 2013;121:4021-4031; quiz 4250.

27. Johnson NA, Slack GW, Savage KJ, et al. Concurrent expression of MYC and BCL2 in diffuse large B-cell lymphoma treated with rituximab plus cyclophosphamide, doxorubicin, vincristine, and prednisone. J Clin Oncol 2012;30:3452-3459.

28. Kiyasu J, Miyoshi H, Hirata A, et al. Expression of programmed cell death ligand 1 is associated with poor overall survival in patients with diffuse large B-cell lymphoma. Blood $2015 ; 126: 2193-2201$.

29. Chen BJ, Chapuy B, Ouyang J, et al. PD-L1 expression is characteristic of a subset of aggressive B-cell lymphomas and virus-associated malignancies. Clin Cancer Res 2013;19:34623473. 
30. Pileri SA, Gaidano G, Zinzani PL, et al. Primary mediastinal B-cell lymphoma: high frequency of BCL-6 mutations and consistent expression of the transcription factors OCT-2, BOB.1, and PU.1 in the absence of immunoglobulins. Am J Pathol 2003;162:243-253.

31. Wilson WH, Pittaluga S, Nicolae A, et al. A prospective study of mediastinal gray-zone lymphoma. Blood 2014;124:1563-1569.

32. Yoon DH, Choi DR, Ahn HJ, et al. Ki-67 expression as a prognostic factor in diffuse large B-cell lymphoma patients treated with rituximab plus CHOP. Eur J Haematol 2010;85:149157.

33. Kluk MJ, Chapuy B, Sinha P, et al. Immunohistochemical detection of MYC-driven diffuse large B-cell lymphomas. PLoS One 2012;7:e33813.

34. Green TM, Young KH, Visco C, et al. Immunohistochemical double-hit score is a strong predictor of outcome in patients with diffuse large B-cell lymphoma treated with rituximab plus cyclophosphamide, doxorubicin, vincristine, and prednisone. J Clin Oncol 2012;30:3460-3467.

35. Alizadeh AA, Eisen MB, Davis RE, et al. Distinct types of diffuse large B-cell lymphoma identified by gene expression profiling. Nature 2000;403:503-511.

36. Chang CC, McClintock S, Cleveland RP, et al. Immunohistochemical expression patterns of germinal center and activation B-cell markers correlate with prognosis in diffuse large B-cell lymphoma. Am J Surg Pathol 2004;28:464-470.

37. Pardoll DM. The blockade of immune checkpoints in cancer immunotherapy. Nat Rev Cancer 2012;12:252-264.

38. Yamamoto W, Nakamura N, Tomita N, et al. Clinicopathological analysis of mediastinal large B-cell lymphoma and classical Hodgkin lymphoma of the mediastinum. Leuk Lymphoma 2013;54:967-972. 
39. Menter T, Bodmer-Haecki A, Dirnhofer S, Tzankov A. Evaluation of the diagnostic and prognostic value of PDL1-expression in Hodgkin- and B-cell lymphomas. Hum Pathol 2016. 40. Yamamoto R, Nishikori M, Kitawaki T, et al. PD-1-PD-1 ligand interaction contributes to immunosuppressive microenvironment of Hodgkin lymphoma. Blood 2008;111:3220-3224. 41. Ansell SM, Lesokhin AM, Borrello I, et al. PD-1 blockade with nivolumab in relapsed or refractory Hodgkin's lymphoma. N Engl J Med 2015;372:311-319.

42. Muenst S, Hoeller S, Willi N, et al. Diagnostic and prognostic utility of PD-1 in B cell lymphomas. Dis Markers 2010;29:47-53.

43. Carreras J, Lopez-Guillermo A, Roncador G, et al. High numbers of tumor-infiltrating programmed cell death 1-positive regulatory lymphocytes are associated with improved overall survival in follicular lymphoma. J Clin Oncol 2009;27:1470-1476.

44. Bachireddy P, Burkhardt UE, Rajasagi M, Wu CJ. Haematological malignancies: at the forefront of immunotherapeutic innovation. Nat Rev Cancer 2015;15:201-215.

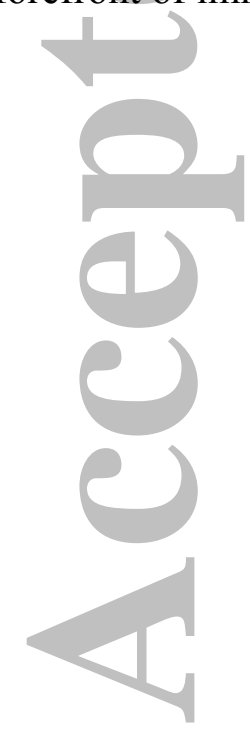




\section{Figure Legends}

Figure 1: Immunophenotypic spectrum and expression of MUM1 and PDL1 in PMBL. AD: An example of PMBL (A, H\&E) with typical immunophenotypic features of high overall expression of CD20 (B), and some expression of CD23 (C) and CD30 (D). E-F: CD15 was weakly or focally expressed in tumor cells of a minority of cases (E and inset) and showed moderate or strong overall staining of many tumor cells in rare cases (F). G-L: Examples of two PMBL cases, one (G, H\&E) showing no/low overall MUM1 staining (H) and high overall PDL1 staining (I), and another (J, H\&E) showing high overall MUM1 staining (K) and no/low overall PDL1 staining (L).

Figure 2: Kaplan-Meier curves of progression-free survival. Association with PDL1 expression (H-score 0-3 vs. 4-12) (A); combined PDL1/MUM1 biologic risk score ranging from 0-2 with one point each for negative or low overall PDL1 (H-score 0-3) and high overall MUM1 (H-score 4-12) (B); R-IPI alone (C); and R-IPI (0-2 vs. >2) with incorporation of PDL1/MUM1 biologic risk score (0-1 vs. 2) (D). Tables below each curve represent number of patients at risk at each time point. 
Table I. Clinical features of PMBL patients*

\begin{tabular}{lc}
\hline Variable & $n$ \\
\hline Age $(n=49)$ & 37 (range 19-82) \\
Median & $5(10 \%)$ \\
Over 60 & $34(69 \%)$ \\
Female gender $(n=49)$ & \\
Ann Arbor Stage $(n=49)$ & $18(37 \%)$ \\
I & $21(43 \%)$ \\
II & $1(2 \%)$ \\
III & $9(18 \%)$ \\
IV & $36(84 \%)$ \\
Elevated LDH ( $n=43)$ & $37(77 \%)$ \\
ECOG performance status 0-1 ( $n=48)$ & \\
R-IPI ( $n=48)$ & $41(85 \%)$ \\
Low to Intermediate (0-2) & $7(15 \%)$ \\
High (>2) & $11($ range $5-20)$ \\
Mediastinal mass median size (cm; $n=42)$ & $29(69 \%)$ \\
Mass size $\geq 10$ cm & $15(33 \%)$ \\
Any non-contiguous extranodal disease $(n=46)$ & $7(16 \%)$ \\
$>1$ non-contiguous extranodal site $(n=45)$ & $6(1-8)$ \\
Median number of R-CHOP cycles & $35(73 \%)$ \\
Consolidative radiation in responding patients $(n=48)$ &
\end{tabular}

\footnotetext{
${ }^{*}$ Clinical and outcome data on a major subset of this cohort have been reported previously. ${ }^{23}$ LDH, lactate dehydrogenase; ECOG, Eastern Cooperative Oncology Group; R-IPI, revised International Prognostic Index; R-CHOP, rituximab, cyclophosphamide, doxorubicin, vincristine, and prednisone.
} 
Table II. Immunohistochemical features of PMBL

\begin{tabular}{|c|c|c|c|c|}
\hline & \multirow[b]{2}{*}{ Antibody } & \multicolumn{2}{|c|}{ Positive, $\mathbf{N}(\%)$} & \multirow{2}{*}{$\begin{array}{c}\text { Negative, } \mathbf{N} \\
(\%)\end{array}$} \\
\hline & & High & Low & \\
\hline \multirow{9}{*}{$\begin{array}{c}\text { Pan-B-cell } \\
\text { markers }\end{array}$} & BOB.1 & $44(92)$ & $2(4)$ & $2(4)$ \\
\hline & CD19 & 45 (94) & $3(6)$ & 0 \\
\hline & CD20 & $47(98)$ & $1(2)$ & 0 \\
\hline & $\mathrm{CD} 22$ & $33(67)$ & $14(29)$ & $2(4)$ \\
\hline & CD23 & $18(37)$ & $14(29)$ & $17(35)$ \\
\hline & CD45 & $47(98)$ & $1(2)$ & 0 \\
\hline & CD79a & $35(73)$ & $12(25)$ & $1(2)$ \\
\hline & Ост2 & $39(81)$ & $7(15)$ & $2(4)$ \\
\hline & PAX5 & $46(96)$ & $1(2)$ & $1(2)$ \\
\hline \multirow{2}{*}{$\begin{array}{l}\text { Hodgkin } \\
\text { markers }\end{array}$} & CD15 & $3(6)$ & $12(25)$ & 33 (69) \\
\hline & CD30 & $19(40)$ & $18(38)$ & $11(23)$ \\
\hline \multirow{9}{*}{$\begin{array}{c}\text { Hans } \\
\text { classifier }\end{array}$} & CD10 & $6(14)$ & $5(12)$ & $32(74)$ \\
\hline & BCL6 & $27(61)$ & $15(34)$ & $2(5)$ \\
\hline & MUM1 & $36(73)$ & $10(20)$ & $3(6)$ \\
\hline & $\mathrm{BCL} 2$ & $28(64)$ & $12(27)$ & $4(9)$ \\
\hline & PDL1 & $20(43)$ & $13(28)$ & $13(28)$ \\
\hline & PD1 & 0 & $2(5)$ & $39(95)$ \\
\hline & & Average & Range & $\geq 40 \%, N(\%)$ \\
\hline & Ki67 (\%) & 32 & $0-100$ & $17(38)$ \\
\hline & MYC (\%) & 28 & $0-70$ & $16(34)$ \\
\hline \multirow{4}{*}{$\begin{array}{l}\text { Cell of } \\
\text { origin }\end{array}$} & \multirow{4}{*}{\multicolumn{2}{|c|}{$\begin{array}{l}\text { Germinal center: } \\
\text { Non-germinal center: } \\
\text { Not evaluable: }\end{array}$}} & $10(23 \%)$ & \\
\hline & & & $33(77 \%)$ & \\
\hline & & & 6 & \\
\hline & & ing: & $6(14 \%)$ & \\
\hline \multirow[t]{2}{*}{ BCL2/MYC } & Non-doub & pressing: & $38(86 \%)$ & \\
\hline & Not evalu & & 5 & \\
\hline
\end{tabular}

Overall score: High or Moderate/Strong/Diffuse (H-score 4-12), Low or Weak/Focal (Hscore 1-3), Negative (H-score 0). PD1 staining of tumor-infiltrating lymphocytes. Cell of origin based on the Hans classifier. ${ }^{25}$ Double-expression defined as $\geq 40 \%$ MYC and $\geq 50 \%$ BCL2 staining. ${ }^{27}$ Total $N$ is less than 49 for some stains because some antibodies were not evaluable for all cases. 

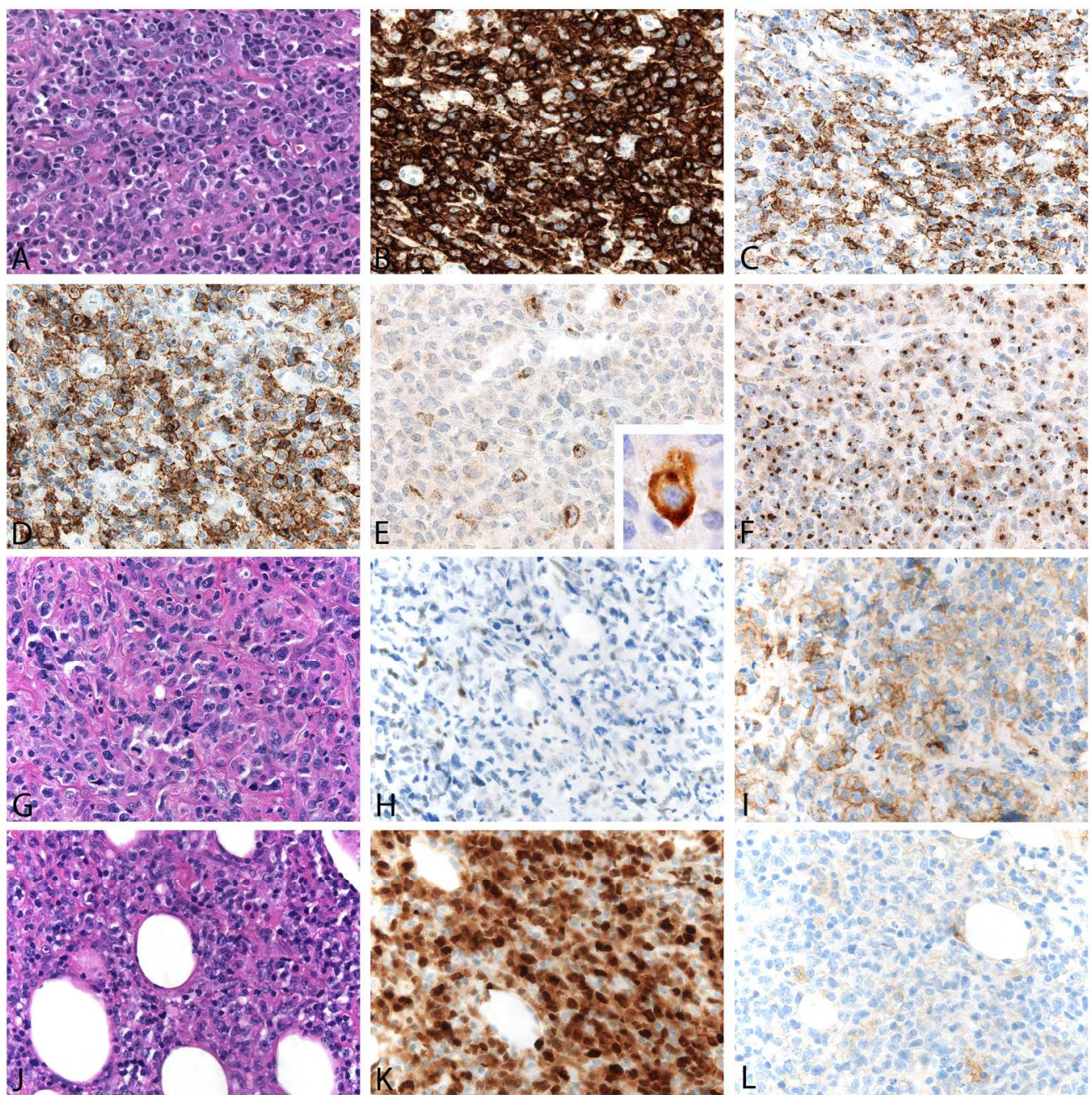

Figure 1: Immunophenotypic spectrum and expression of MUM1 and PDL1 in PMBL. A-D: An example of PMBL (A, H\&E) with typical immunophenotypic features of high overall expression of CD20 (B), and some

expression of CD23 (C) and CD30 (D). E-F: CD15 was weakly or focally expressed in tumor cells of a minority of cases ( $E$ and inset) and showed moderate or strong overall staining of many tumor cells in rare cases (F). G-L: Examples of two PMBL cases, one (G, H\&E) showing no/low overall MUM1 staining $(H)$ and high overall PDL1 staining (I), and another (J, H\&E) showing high overall MUM1 staining (K) and no/low overall PDL1 staining (L). $185 \times 185 \mathrm{~mm}(300 \times 300 \mathrm{DPI})$ 

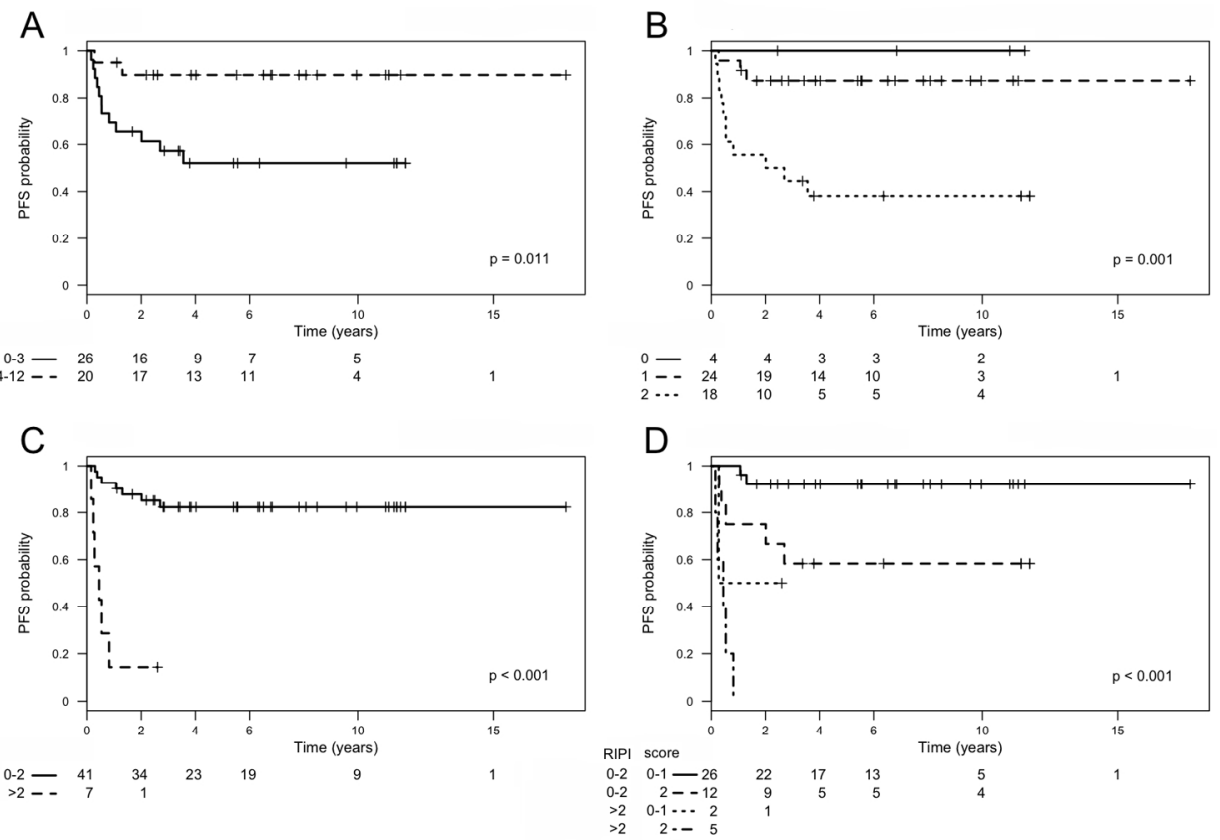

Figure 2: Kaplan-Meier curves of progression-free survival. Association with PDL1 expression (H-score 0-3 vs. 4-12) (A); combined PDL1/MUM1 biologic risk score ranging from 0-2 with one point each for negative or low overall PDL1 (H-score 0-3) and high overall MUM1 (H-score 4-12) (B); R-IPI alone (C); and R-IPI (0-2 vs. >2) with incorporation of PDL1/MUM1 biologic risk score (0-1 vs. 2) (D). Tables below each curve represent number of patients at risk at each time point. $635 \times 423 \mathrm{~mm}(72 \times 72 \mathrm{DPI})$

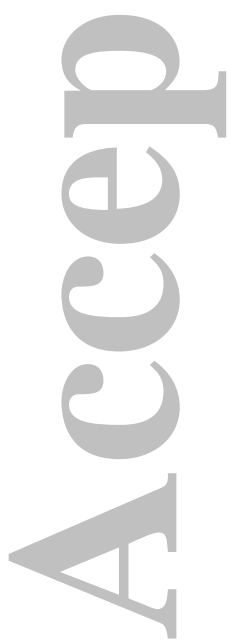

